\title{
Simulating Leakage Impact on Steel Industrial System Functionality
}

\author{
Mohamad Pazlin bin Saion \\ Faculty of Manufacturing Technical University of Malaysia Malacca \\ P051910004@student.utem.edu.my \\ https://orcid.org/0000-0001-8528-5057
}

\begin{tabular}{|c|c|}
\hline Article History & Abstract \\
\hline $\begin{array}{l}\text { Article Submission } \\
21 \text { April } 2021 \\
\text { Revised Submission } \\
18 \text { June } 2021 \\
\text { Article Accepted } \\
22 \text { July } 2021 \\
\text { Article Published } \\
30 \text { September } 2021\end{array}$ & $\begin{array}{l}\text { Steels exhibiting ultralow sulfur content tend to gain increasing usage or application in } \\
\text { manufacturing pipes used in oil transportation, as well as offshore platform } \\
\text { construction. For these systems or platforms, it is worth indicating that they call for } \\
\text { high resistance and impact strength relative to the formation of lamellar cracks. In this } \\
\text { study, it was documented that when a refinery is compromised, the resultant fluids that } \\
\text { are released tend to cause a loss in the refinery's transportation capability. This } \\
\text { outcome suggests that there is likely to be a decrease in the efficiency of transmission. } \\
\text { Notably, the study examined the extent to which a leak presence affects flow behavior } \\
\text { in the context of annular mist flow regime's deep water production flow line. Another } \\
\text { context that was investigated involved slug and stratified flow regimes. From the } \\
\text { results, given the slug and annular flow regimes, any leakage could alter the pattern of } \\
\text { material flow. } \\
\text { Keywords: Manufacturing, Offshore Platform Construction, Steel Industry }\end{array}$ \\
\hline
\end{tabular}

\section{Introduction}

Given multi-methods such as Hydraulic Grade Line (HGL) and Wald's Sequential Probability Ratio Test (SPRT), a CFD simulation system (LDS) is proposed. The system entails wireless communication devices and data acquisition devices. Some of the calculations involved include the positioning module, the pattern recognition module, the CFD simulation module, and the data processing module. From the single-phase gas medium's simulation and test outcomes, a detection system coupled with SPRT and other techniques yields good performance. Indeed, there is only $1 \%$ difference in leak location accuracy, should the refinery be adequately long. For small leaks that are less than $5 \%$, their detection and alarming takes about 30 minutes. Also, less than $18 \%$ of the error of the rate of leakage is reported $[1,2]$.

With a leak as a source term, a single-pressure two-fluid flow has been established by [3-5], with mass balance equations on the focus. The model has been proposed to simulate a stratified-pattern two-phase flow, considering a horizontal pipe prone to leakage. From the results, it is evident that relative to the intersection of the pressure profile downstream and upstream of the given leak, the leak localization technique could be extended to gain application in two-phase flow refineries (from single-phase flow refineries), especially in stratified slow patterns. Indeed, the latter pattern dominates many production gas refineries.

\section{Methodology}

Relative to the Kantorovich distance concept, a novel data-driven leak localization and detection algorithm was proposed. In the Kantorovich distance (KD), the main concept entails the minimum cost or distance through which the mass could be trans-located from one probability distribution to another. To detect potential alterations in the status of the refinery, major parameters employed include pressure values and mass flow rates. Relative to the 
signature of the refinery leak, there is CFD simulation, hence location determination. Indeed, this framework was applied in transient conditions, having simulated a refinery. To prove the model's efficacy, it was applied to an industrial refinery network in real-time controlled leak tests. Indeed, the technique was able to detect large and small ( $1 \%$ of the nominal rate of flow) test leaks within the given real refinery. Also, there was a significant reduction in the time required to detect and locate the leaks, compared to the case of commercial CFD simulation systems. Overall, the proposed model for leak localization was discerned to be accurate, especially in situations involving small leaks [7]. Although, it was mentioned that the detection time and localization of the proposed method was better existing commercial LDS techniques, the methods were not specified.

\section{Results and Discussion}

In relation to the particle filter, it is notable that it reflects a consequential Monte Carlo technique influenced by the point mass variable, whereby the state in both the nonlinear systems and non-Gaussian systems can be estimated by using the probability density functions without demanding conducting the linearization process. Also, research has demonstrated how, by applying the adaptive particle filter model, faults can be detected and isolated for an algorithm in which there is a need for a time-varying parameter estimation approach. Also, the model can aid in small CFD simulation.

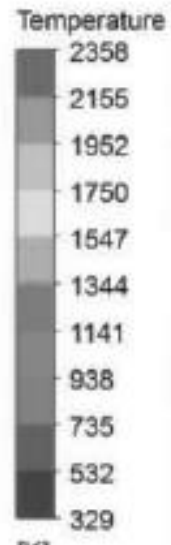

[K]

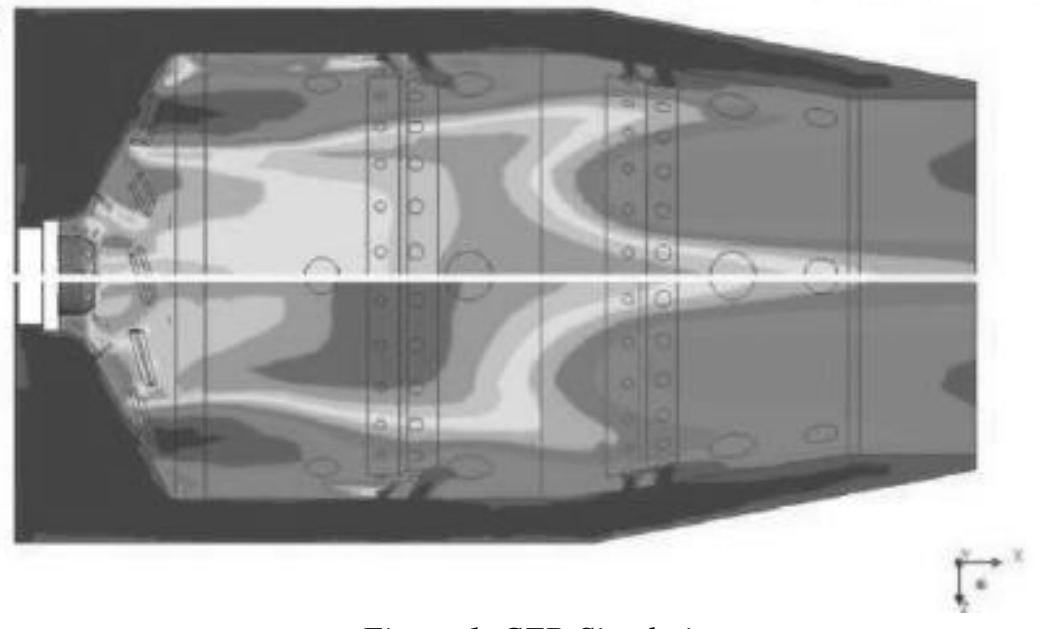

Figure 1. CFD Simulation
$100 \% \mathrm{CH}_{4}$

$100 \% \mathrm{H}_{2}$

A combination of approaches has been proposed namely, extended real time transient modeling (E-RTTM). The model is used to create a real time reference for the flow measurement to eliminate transient conditions in the first step. The results from the model are analyzed separately and in sum to identify short term variations. This step is scanning for typical footprints of leaks and is based on pattern recognition. Because of this any alarm will be triggered in the pattern recognition tool instead inside the model. This is eliminating the sensitivity of the model against environmental effects and makes is long term stable without facing the disadvantage of a permanent maintaining process of the model. For the Real Time Transient Monitoring (RTTM), the statistical volume balance technique was used to replace it to ensure that leaks are detected up to $0.5 \%$, even when transient conditions set in without yielding false alarms in the course of events such as during switchovers, temperature changes, shut down or start up, crude oil changes, and pump switchover [8]. For the case of multiphase flow lines, especially those that rely on the mass balance approaches, they fail to detect and localize leaks. However, the PPA and RTTM approaches can be seen to be able to exhibit sufficient performance in the context of multiphase flow lines. Concerning the compensated mass balance technique, it has been assessed previously relative to its capacity to detect leaks. The selected approach is able to handle various refinery operating conditions and detect leakages from other abnormal states of the operating conditions. Given that the transient framework of this approach is based or relies on factors of 
density, temperature, pressure, and flow rate towards the calculation of the packing rate of the refinery, the compensated mass balance approach or framework is able to handle various operational alterations of the refinery, allowing further for the easy detection of the leak. Different researchers have evaluated different outputs with respect to CFD simulation. However, from the literature it is obvious that flow rate is the main factor while identifying a leak. Hence, most of the researchers have focused on flow rate varying time.

The term diagnostics is largely got confused with detection. Fault detection is simply the indication or alarm of fault. Usually, fault is detected first which triggers diagnostics module or algorithm to; identify the type, point, size, and time of fault. In the case of fault diagnostics in refinery, mostly leak size and leak location are estimated. For leak diagnostics, one of the approaches entails the utilization of equation-generated binary signatures. Another alternative that can be used in the place of binary diagnostics involves direct diagnostician or fuzzy diagnostics via the implementation of tools in the form of ANN (artificial neural networks). Hardly, the fuzzy idea tends to be discussed in the refinery context, especially when it comes to gas refineries. Thus, when fuzzy systems are used, it is projected that the field of refinery leak diagnostics might yield promising outcomes concerning the prediction of the refinery's various operating conditions. For leak localization, the process entails establishing leak points in short refineries. In case of long refineries, leak localization refers to the identification of leak or faulty sections. For the PGM and NPW, they reflect classical techniques through which leak point estimation is achieved. For this method, however, the main problems are that the determination of leak localization requires the propagated artificial wave that the valve closure generates, yet this activity's application comes with much difficulty in actual refineries. The challenge is attributed to the stoppage of the fluid supply within the refinery. From previous reviews, the NPW, relative to fault detection, is not appropriate for long refineries due to its sensitivity to transients, which could cause false alarm incidence. In the refinery, also, pressure alterations are not only linked to leakages. Rather, they are attributed to temperature alterations, as well as variations in the rate of flow and associated disruptions. Hence, the need for the same criterion towards the determination of process disruptions with the leak could not be overstated.

It can be observed further that when leak occurred pressure gradient before and after the leakage is changed and their intersection is indication a leak location. In the study of oil refinery leak location used highly precise pressure sensors at refinery ends and estimated leak location with an error of $\pm 200 \mathrm{~m}$. As it can be concluded that for steady state conditions PGM is detection a leakage with big errors. So, for transient cases with process noise it is very difficult to find good estimated of leak position with PGM.

Prediction of the flow pattern is essential to prediction of pressure gradient within the longitudinal refinery length and, therefore, the behavior of phases within the flow conduit. Computational and physical refinery challenges are linked with these variables, including prediction of mass exchange between phases, vibration issues, wear, and corrosion [18]. These factors must be accounted for in any refinery-management program, particularly for refineryleak monitoring and prediction.

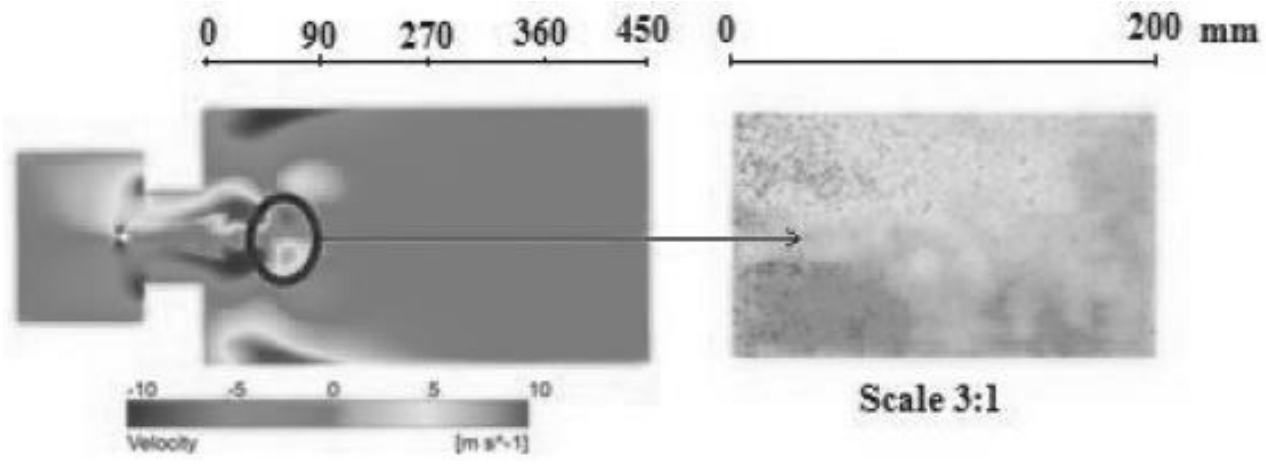

Figure 2. PGM Detection 
For the case of acoustic pressure waves, it is notable that they travel in refineries at a speed similar to that of the fluid sound, especially for the case of fluids under transportation. Through dynamic pressure sensors, the pressure waves can be detected. Indeed, different models and filters can aid in the determination of this disruption and also ensure that it is differentiated from other refinery's pressure events. The architecture, indeed, can be used also for signal and noise pattern recognition, eventually paving the way for potential leak event auto-alarming. Furthermore, the respective manufacturers of these technologies use unique frameworks or processing techniques towards capturing and analyzing pressure signals responsible for the future prediction of leaks and leak locations. Indeed, several techniques of leak localization and CFD simulation have been employed, whether exterior or interior. Imperatively, all the approaches exhibit their drawbacks and strengths. Four main characteristic features such as diagnostics, transient conditions, multiphase and performance measures have been categorised. Based on the review so far, the LDS methods can be selected based on various factors, such as, accuracy, complexity, training data requirement and cost of installation. The complexity of these methods increases from experience-based methods to model based methods. The amount of training data required for satisfying results decreases from experience-based methods to model based methods. The accuracy of these methods may vary from technique to technique; hence it is categorised into very low, low, high, and very high categories. There is a contradiction in literature point of view for cost of installation for various methods. The mass balance method is considered to be least expensive among all by few researchers [9-13], whereas it is considered as highly expensive in others' works [1]. Similarly acoustic technique is considered as high cost by a researcher [12] and medium by few researchers [13-15].

To get the maximum output of the available techniques, different methodologies and technologies may be combined. The NPW method can give accurate leak locations, quick detection time for unanticipated leaks while the highly reliable statistical analysis can supplement it, which has the capability of detecting slowly developing leaks. In many cases the statistical analysis has been combined with RTTM, which helped in reducing the false alarms. Various aspects like false alarms, response time and localization of leaks are discussed here. Since there are many probabilities and scenarios for occurring of leakages, there is a necessary to look in all the possible aspects to reduce the damage. The refineries can be highly subjected to multiple leaks; hence, we have to check for a CFD simulation system which can detect multiple leaks. In some cases, leaks can be tiny, but the damage is not negligible, so CFD simulation systems which can detect small leaks have to be employed. One of the most important parameters is false alarms, which cannot be ignored. The efficiency of the CFD simulation system can be evaluated based on false alarms.

\section{Conclusion}

Overall, the best operational conditions in the refinery process are better placed to allow for productivity improvement when it comes to the removal of material such as sulfur in secondary and primary processes, metallurgy included.

\section{References}

[1] Haroun Y., Raynal L. (2015) Use of Computational Fluid Dynamics for absorption packed column design, Oil Gas Sci. Technol.

[2] Solomenko Z., HarounY., FouratiM., Larachi F., Boyer C.,Augier F. (2015) Liquid spreading in trickle-bed reactors: Experiments and numerical simulations using Eulerian-Eulerian two-fluid approach, Chem. Eng. Sci. $126,698-710$

[3] Abedini E, Zarei T, Rajabnia H, Kalbasi R, Afrand M (2017) Numerical investigation of vapor volume fraction in subcooled flow boiling of a nanofluid. J Mol Liq 238:281-289

[4] Hosseini S, Fattahi M, Ahmadi G (2017) Investigation of hydrodynamics and heat transfer in pseudo 2D spouted beds with and without draft plates. Braz J Chem Eng 34:997-1009

[5] Oliveira F, Martignoni W, Souza E, Pereira L, Santos L, Prata D, Monteiro L (2017) Convective recirculation effect on the selective non-catalytic reduction behavior in an industrial furnace. Braz $\mathrm{J}$ Chem Eng 34:1011-1021 